- The use of postal reminders for orthodontic consultation appointments appears to result in a useful increase of appointments that are kept or cancelled in advance

- If a patient receives a reminder and does not return the confirmation slip, there is a $33 \%$ chance that they will not attend.

- The use of a reminder does not counteract the effect of social deprivation on non-attendance at the clinic.

\title{
The use of postal reminders to reduce non-attendance at an orthodontic clinic: A randomised controlled trial
}

\author{
S. Can, ${ }^{1}$ T. Macfarlane ${ }^{2}$ and K. D. O'Brien ${ }^{3}$
}

Objectives To evaluate the effect of issuing a patient reminder plus a confirmation slip on the attendance of orthodontic new patients. Setting Department of Orthodontics, University Dental Hospital of Manchester.

Design A randomised controlled trial.

Method New patients were randomly allocated to:

i) receive a reminder letter and return a confirmation slip or

ii) not receive a reminder.

Outcome measures Patient attendance at the clinic.

Results A total of 232 patients were entered into the study between June 18, 2001 and August 29, 2001. These were randomly allocated to $115(49.8 \%)$ in the reminder group and 116 (50.2\%) in the no reminder group. If the patient received a reminder and returned the confirmation they were less likely to fail the appointment than if they did not receive a reminder ( $\mathrm{OR} 0.4,95 \% \mathrm{Cl} 0.2$ to 0.96 ) There was an effect of social deprivation, if the patients lived in an area of high social deprivation they were 2.7 ( $95 \% \mathrm{Cl} 1.1$ to 6.5 ) times more likely to fail to attend an appointment than people who were more affluent.

Conclusions The use of postal reminders for orthodontic consultation appointments appears to result in a useful increase of appointments that are kept or cancelled in advance.

This paper describes a randomised trial that evaluated the effectiveness of a simple postal reminder system on failure to attend appointments in a secondary care orthodontic clinic.

Patient non-attendance is an area of concern for all providers of healthcare, as it incurs costs and results in a loss of clinical time. When we consider the secondary care sector for orthodontic consultation appointments, recent investigations have revealed that non-attendance is as high as $19.4 \% .{ }^{1}$ The cause of this problem has been investigated in several studies. For example, when

${ }^{1}$ Postgraduate Student in Orthodontics, ${ }^{2}$ Lecturer in Dental Statistics, ${ }^{3}$ Professor of Orthodontics, Department of Dental Medicine and Surgery, University Dental Hospital of Manchester, Higher Cambridge Street, Manchester M15 6FH

Correspondence to: Prof. Kevin O'Brien

E-mail:Kevin.O'Brien@man.ac.uk

\section{Refereed pape}

Received 09.12.02; Accepted 18.03.03

doi:10.1038/sj.bdj.4810443

(c) British Dental Journal 2003; 195: 199-201 the reasons for failed appointments in the University Orthodontic Department in Belfast were analysed, ${ }^{2}$ they concluded that the most frequent reasons for failure were illness, forgetfulness, transport difficulties and other commitments. These other commitments usually involved schoolwork in the form of examinations, field trips or sporting activities.

In another investigation Trenouth and Hough investigated the reasons for broken and cancelled appointments in a district general hospital orthodontic clinic. They also concluded that forgetfulness was the most common reason for missing an appointment, while illness was the most common reason for cancelling. ${ }^{3}$

Another important factor that may influence attendance is socio-economic status. For example some patients who fail their appointment suggested that it was difficult to take time off work, in addition to having problems travelling to the clinic. ${ }^{2,3}$

In view of these studies, one solution to this problem may be using postal or telephone reminders. Several studies have been carried out into this area.

Postal reminders were investigated by Woolgrove et al., and they developed a personalised calendar sheet, that contained a hand written message. ${ }^{4}$ They divided 938 patients into two groups using alternate addresses on computer files. One group was sent the reminder and the other was sent a standard appointment card. They found that the use of the reminder resulted in an increase in the number of appointments kept, from 50\% to 60\%, and a decrease in failed appointments, from $33 \%$ to $27 \%$.

In a more extensive clinical study, the effect of four reminder systems in a single handed general dental practice were evaluated..$^{5}$ A total of 2,500 patients were allocated to one of five groups. These were: (i) no reminder, (ii) postal reminder, (iii) manual telephone call, (iv) automated telephone call, and (v) automated telephone plus reminder. They found that there was a reduction in the failed attendance rate from 9.4\% (no reminder) to a minimum of $3 \%$ by reminding patients, with any method. However, there was no significant difference among the four reminder groups.

We can, therefore, conclude that the use of a reminder does result in a reduction in failed appointments. However, these studies were carried out in the primary care sector and the findings may not be relevant to secondary care clinics in hospitals. This was the aim of our investigation. 


\section{Null hypothesis}

We tested the following null hypotheses:

- There is no effect of a postal reminder on non-attendance at a dental hospital orthodontic consultation clinic.

- Socio-economic status or gender of the patients does not influence the effect of a postal reminder on non-attendance.

\section{MATERIAL AND METHODS}

\section{Protocol}

All patients who were due to be sent an appointment for a new patient consultation in the Orthodontic Unit of the University Dental Hospital of Manchester over a 10-week period from the 18 June 2001 were eligible for inclusion in the study. They were randomly allocated using a random number table to either receive an appointment or to receive an appointment plus a reminder that was sent at least two weeks before their appointment. The reminder letter was sent with a stamped addressed postcard, which we asked the patient to return, so that the appointment could be confirmed.

We also obtained details of the patient's socio-economic status expressed as the Townsend index from their postcode. ${ }^{6}$

The outcome measure for this study was whether the patient attended the clinic. As the investigators could not influence this outcome, we did not use a blinding strategy.

The sample size for this investigation was calculated using the results of a previous investigation into attendance at the same department. We decided that a meaningful reduction in non-attendance was $15 \%$. To have a study with a power of 0.80 and an alpha level of 0.05 we needed to enroll 100 patients into each group. ${ }^{1}$

The data were analysed with the chi-squared test and logistic regression to evaluate the influence of (i) the reminder, (ii) whether the patient returned the confirmation card and (iii) socio-economic status and gender on the dependent variable of non-attendance.

\section{RESULTS}

A total of 232 patients were entered into the study between June 18, 2001 and August 29, 2001. These were randomly allocated into $115(49.8 \%)$ in the reminder group and 116 (50.2\%) into the no reminder group.

Table 1 contains the basic descriptive data for the study. Data analysis with the chi-squared statistic revealed that the reminders appeared to have an effect on attendance/cancellation of the patients $(P=0.001)$ and patients from areas of higher social deprivation were less likely to attend/ $(P=0.039)$. The results of the logistic regression analysis on the dependant variable of attendance are presented in Table 2. This reveals that failure of appointment was influenced by all three factors in the model:

1. The gender of the patient,

2. If they received a reminder and returned a confirmation

3. The level of social deprivation of the area in which the patient lived.

In summary, it appears that girls were twice as likely to fail an appointment than boys. If patients received a reminder and returned the confirmation they were less likely to fail the appointment than if they did not receive a reminder (OR 0.4, 95\% CI 0.2 to 0.96) Finally, there was an effect of social deprivation, if the patients lived in an area of high social deprivation they were 2.7 times (95\% CI 1.1 to 6.5) more likely to fail an appointment than people who were more affluent.

\section{DISCUSSION}

The most relevant finding of this investigation was that if people was sent a reminder postcard for their orthodontic consultation and they returned the confirmation slip, they were almost twice as likely to attend their appointment than if they did not receive a reminder. This has some implications for the provision of orthodontic new patient cancellations. We could suggest that if nonattendance is a problem at a clinic, the introduction of a system of reminders may result in a meaningful increase in effectiveness of the clinic. For example, if a the target attendance for a clinic is 20 patients, then the consultant has the following choices:

- Assume an attendance rate of 65\% and overbook the clinic by seven patients and take the risk of all the patients attending and consequent disruption to the clinic.

- Issue a reminder and request a confirmation by postcard. This should result in an attendance rate of $83 \%$. If the patient does not return the confirmation slip there is only a $48 \%$ chance of the patient attending and consideration could be given to sending for additional patients to compensated for the predicted shortfall.

\begin{tabular}{|c|c|c|c|c|c|}
\hline \multirow[t]{2}{*}{ Factor } & & \multicolumn{4}{|c|}{ Attendance at clinic } \\
\hline & & Yes & No & Cancelled & Total \\
\hline \multirow[t]{3}{*}{ Reminder } & $\begin{array}{l}\text { Reminder } \\
\text { and reminder } \\
\text { returned }\end{array}$ & $\begin{array}{c}68 \\
(82.9 \%)\end{array}$ & $\begin{array}{c}10 \\
(12.2 \%)\end{array}$ & $\begin{array}{c}4 \\
(4.9 \%)\end{array}$ & 82 \\
\hline & $\begin{array}{l}\text { Reminder } \\
\text { and } \\
\text { reminder } \\
\text { not returned }\end{array}$ & $\begin{array}{c}16 \\
(48.5 \%)\end{array}$ & $\begin{array}{c}12 \\
(33.3 \%)\end{array}$ & $\begin{array}{c}6 \\
(18.2 \%)\end{array}$ & 33 \\
\hline & No reminder & $\begin{array}{c}75 \\
(64.7 \%)\end{array}$ & $\begin{array}{c}27 \\
(23.3 \%)\end{array}$ & $\begin{array}{c}14 \\
(12.1 \%)\end{array}$ & 116 \\
\hline \multirow[t]{2}{*}{ Gender } & Male & $\begin{array}{c}79 \\
(71.8 \%)\end{array}$ & $\begin{array}{c}17 \\
(15.5 \%)\end{array}$ & $\begin{array}{c}14 \\
(12.7 \%)\end{array}$ & 110 \\
\hline & Female & $\begin{array}{c}80 \\
(66.1 \%)\end{array}$ & $\begin{array}{c}31 \\
(25.6 \%) \\
\end{array}$ & $\begin{array}{c}10 \\
(10.3 \%)\end{array}$ & 121 \\
\hline \multirow{3}{*}{$\begin{array}{l}\text { Social } \\
\text { deprivation } \\
\text { (Townsend } \\
\text { index) }\end{array}$} & $\begin{array}{l}\text { Low } \\
<-2.05\end{array}$ & $\begin{array}{c}61 \\
(78.2 \%)\end{array}$ & $\begin{array}{c}9 \\
(11.5 \%)\end{array}$ & $\begin{array}{c}8 \\
(10.3 \%)\end{array}$ & 78 \\
\hline & $\begin{array}{l}\text { Medium } \\
2.05-7.52\end{array}$ & $\begin{array}{c}53 \\
(68.8 \%)\end{array}$ & $\begin{array}{c}17 \\
(22.1 \%)\end{array}$ & $\begin{array}{c}7 \\
(9.1 \%)\end{array}$ & 77 \\
\hline & $\begin{array}{l}\text { High } \\
>7.85\end{array}$ & $\begin{array}{c}45 \\
(59.2 \%)\end{array}$ & $\begin{array}{c}22 \\
(28.9 \%)\end{array}$ & $\begin{array}{c}9 \\
(11.8 \%)\end{array}$ & 76 \\
\hline
\end{tabular}


Table 2 Logistic regression to evaluate the influence of the reminder, return of the reminder and social deprivation on non-attendance at the clinic

Factor Odds ratio $\quad 95 \% \mathrm{Cl}$ for odds ratio

Lower

Upper

\section{Gender}

Male

Female

Reminder

No reminder

Reminder sent

and returned

Reminder sent

but not returned

Socio-economic

deprivation

(Townsend)

Low

Medium

High

1.0
2.4

2.4
2.71

2.03

1.02

4.06

1.00

0.43

0.19

0.96

1.63

0.67

3.95

While the first option may seem to be the most simple, there is the potential problem that may arise if all the patients attend the clinic. This will result in an overbooked clinic and the possibility that the quality of care may suffer. The second option will lead to additional work by reception staff in monitoring the return of the confirmation slips and booking additional appointments.

It was interesting that we found that girls were more likely to fail an appointment than boys. It is difficult to put forward reasons for this finding. We can suggest that dentists may be more willing to refer a girl when the malocclusion is mild and following the referral the patients then change their mind about attending. This, however, is purely conjecture.
Finally, when the effects of social deprivation are considered, our previous investigation revealed that social deprivation had a negative effect on attendance at the clinic. ${ }^{1}$ This was also found in this investigation, and importantly, the effect of social deprivation was still evident after adjusting for other variables. This confirms that if a clinic has a catchment area in which there is a high level of social deprivation then the use of reminders will not be as effective as in more affluent areas. This also suggests that the main reasons for non-attendance of people who live in deprived areas are more complex than simply forgetting an appointment.

\section{CONCLUSIONS}

- The use of postal reminders for orthodontic consultation appointments appears to result in a useful increase of appointments that are kept or cancelled in advance.

- If patients receive a reminder and do not return the confirmation slip, there is a $33 \%$ chance that they will not attend.

- The use of a reminder does not counteract the effect of social deprivation on non-attendance at the clinic.

The authors would like to acknowledge the help Jackie Kitchen of Central Manchester Health Care Trust.

1. O'Brien K D, Mattick R, Mandall N, Wright J, Conboy F, Gosden T. Are specialist outreach clinics for orthodontic consultation effective? A randomized controlled trial. Br Dent J 2001; 191: 203-207.

2. Richardson A. Failed appointments in an academic orthodontic clinic. Br Dent J 1998; 184: 612-615.

3. Trenouth M J, Hough A. Reasons for broken and cancelled appointments in a British orthodontic clinic. J Clin Orth 1991; 25: 115-120.

4. Woolgrove J, Gelbier S, Cumberbatch $\mathrm{G}$. Increasing dental attendance by using personalized reminders. Comm Dent Health 1988; 5: 389-393.

5. Reekie $\mathrm{D}$, Devlin $\mathrm{H}$. Preventing failed appointments in general dental practice: comparison of reminder methods. Br Dent J 1998; 185: 472-474.

6. Poulsom K. Evaluation of a postal reminder system for the attendance of patients Comm Dent Health 1991; 8: 179-180 